\title{
Susceptibilidad a betalactámicos y resistencia por betalactamasas de espectro extendido (BLEE) en Enterobacteriaceae aisladas de reservorios ambientales de un hospital general en Cajamarca, Perú.*
}

\author{
Beta-lactam susceptibility and resistance by extended spectrum beta-lactamases (ESBL) in \\ Enterobacteriaceae environmental isolations at a general hospital in Cajamarca, Peru. \\ Marco Rivera-Jacinto ${ }^{1}$, Claudia Rodríguez-Ulloa², Gladys Huayán-Dávila ${ }^{3}$, Pedro Mercado-Martínez ${ }^{4}$
}

\section{RESUMEN}

Objetivos: Identificar enterobacterias en reservorios intrahospitalarios, evaluar su sensibilidad a betalactámicos y determinar su capacidad de producir betalactamasas de espectro extendido (BLEE), en el Hospital Regional de Cajamarca. Material y métodos: Se obtuvieron muestras mediante hisopado de grifos, lavatorios, mesas, camas y tablillas de historia clínica en las áreas de cirugía y pediatría; se recuperaron, aislaron e identificaron 45 cultivos de importancia clínica: 14 Enterobacter cloacae, 11 Escherichia coli, 5 Citrobacter freundii, 4 Klebsiella pneumoniae y 11 de otros géneros. Se determinó la sensibilidad antimicrobiana a los antibióticos betalactámicos: ampicilina, cefalotina, cefoxitina, ceftazidima, cefotaxima, ceftriaxona, amoxicilina-clavulanato, aztreonam e imipenem. Teniendo en cuenta los diámetros críticos de tamizaje, se realizó el test confirmatorio para BLEE. Para el análisis estadístico se utilizó el programa estadístico SPSS v15. Resultados: De los 45 cultivos aislados 34 fueron resistentes a ampicilina, 34 a cefalotina, 14 a cefoxitina, 12 a cefotaxima, 11 a ceftriaxona, 5 a ceftazidima, 19 a amoxicilina-clavulanato y 15 a aztreonam. Doce cultivos presentaron resistencia por BLEE a cefalosporinas de tercera generación y/o monobactámicos, cuatro E. coli y cuatro E. cloacae fueron los más relevantes. Todos fueron sensibles a imipenem. Conclusiones: Dada la capacidad de algunos de estos cultivos de producir BLEE existe el riesgo de brotes intrahospitalarios que pueden complicarse cuando son originados por microorganismos multirresistentes. La producción de BLEE como mecanismo de resistencia en los cultivos hallados debe ser motivo de preocupación por las implicancias que tiene.(Rev Med Hered 2011;22:69-75).

PALABRAS CLAVE: Enterobacteriaceae, beta-Lactamasas, farmacorresistencia, infección hospitalaria.

* Estudio presentado en el Primer Encuentro Científico Internacional del Norte Invierno-2010 (ECI-Norte-2010) en la ciudad de Trujillo-Perú en agosto del 2010.

Biólogo-Microbiólogo. Maestro en Ciencias. Laboratorio de Microbiología y Parasitología, Universidad Nacional de Cajamarca., Cajamarca, Perú.

2 Bióloga-Microbióloga. Maestra en Salud Pública. Laboratorio de Microbiología y Parasitología, Universidad Nacional de Cajamarca. Cajamarca, Perú.

Bióloga-Microbióloga. Hospital Regional de Cajamarca. Cajamarca - Perú

Biólogo-Microbiólogo. Doctor en Ciencias Biomédicas. Universidad Nacional de Trujillo. Trujillo - Perú. 


\section{SUMMARY}

Objectives: To identify enterobacteria cultures in nosocomial reservoirs, assess their sensitivity to beta-lactams and their ability to produce extended-spectrum beta-lactamases (ESBLs) at the Hospital Regional de Cajamarca. Material and methods: Samples were collected by cotton swab in faucets, sinks, tables, beds and patients' medical charts in the areas of surgery and pediatrics, 45 cultures clinically important were recovered, isolated and identified: 14 Enterobacter cloacae, 11 Escherichia coli, 5 Citrobacter freundii, 4 Klebsiella pneumoniae and 11 the other genres. An antimicrobial susceptibility test was performed for beta-lactamic antibiotics: ampicillin, cephalothin, cefoxitin, ceftazidime, cefotaxime, ceftriaxone, amoxicillin-clavulanate, aztreonam and imipenem. Given the critical diameters of screening, the confirmatory test for ESBL was performed. For statistical analysis, were used SPSS v15. Results: Of the 45 cultures isolate, 34 were resistant to ampicillin, 34 to cephalothin, 14 to cefoxitin, 12 to cefotaxime, 11 to ceftriaxone, 5 to ceftazidime, 19 to amoxicillin-clavulanate and 15 to aztreonam. Twelve cultures were resistant to third-generation cephalosporins and monobactams for ESBLs, four E. coli and four E. cloacae were the most relevant. All were sensitive to imipenem. Conclusions: Given the ability of some of these cultures to produce ESBL, there is a risk of nosocomial outbreaks that can be complicated when are caused by multiresistant organisms. ESBL production as a resistance mechanism in cultures found cause concern.(Rev Med Hered 2011;22:69-75).

KEYWORDS: Enterobacteriaceae, Beta-lactamases, drug resistance, cross infection.

\section{INTRODUCCIÓN}

La familia Enterobacteriaceae constituye el grupo más grande y heterogéneo de bacilos gramnegativos de importancia médica y son las bacterias que se recuperan con mayor frecuencia en muestras clínicas humanas (1). En las últimas décadas, estos bacilos han adquirido mayor importancia como agentes causantes de infecciones intrahospitalarias (IIH), debido a que han desarrollado fenotipos de resistencia bastante amplios frente a los antimicrobianos (2); situación que ha sido reportada en prácticamente todo el mundo $(3,4)$.

Los betalactámicos son los antimicrobianos de mayor prescripción a nivel intrahospitalario, paralelamente hay un incesante aumento de la resistencia bacteriana (5). La prevalencia de resistencia entre las enterobacterias es alta en todo el mundo y la producción de betalactamasa de espectro extendido (BLEE) constituye el mecanismo más importante y un serio problema que afecta el uso de varios betalactámicos incluyendo cefalosporinas de tercera generación $(\mathrm{C} 3 \mathrm{G})$ (4). Además, los genes que codifican estas enzimas pueden ser trasmisibles por plásmidos y por lo general se acompañan de co-resistencias frente a otros antibióticos, limitando las opciones terapéuticas.

Múltiples estudios indican que el amplio, inadecuado y muchas veces irracional uso de antimicrobianos, así como la falta de programas integrales de vigilancia y control, son causas de la selección de bacterias resistentes siendo las personas más expuestas las que están en los hospitales, lo que puede empeorar su pronóstico e incrementar los costos de atención incluyendo un mayor tiempo de estancia hospitalaria (6). Estas bacterias bien pueden estar contenidas en reservorios hospitalarios, contribuyendo al aumento de las IIH.

Puesto que la mayor parte de investigaciones de resistencia bacteriana se realizan en cultivos de fluidos corporales, las bacterias contenidas en reservorios intrahospitalarios (fómites, dispositivos de uso común o superficies ambientales) son subestimadas en su posible papel de patógenos (7), subestimando su capacidad de portar genes de resistencia y su capacidad de trasmisión. Sin embargo, algunas superficies y elementos de uso hospitalario común han sido reconocidos como reservorios bacterianos con importante potencial de infección: sábanas, camas, barandas de camas, manijas de puertas, mesas, tablillas de historia clínica y otras superficies del entorno inmediato de los pacientes $(8,9)$, sustentando la tesis de que estas bacterias juegan un papel importante en la diseminación de la resistencia y pueden ser determinantes en las IIH, sobre todo porque muchas especies sobreviven por meses sobre superficies inanimadas (10) y porque las manos colonizadas del personal también pueden diseminar estas bacterias, luego del contacto con superficies contaminadas $(8,11)$. Esto nos hace reflexionar sobre la importancia de conocer la epidemiología de estas bacterias para plantear estrategias encaminadas a disminuir y controlar la 
resistencia intrahospitalaria.

En nuestro país, pese a la alta incidencia de IIH, prácticamente no existen reportes de mecanismos de resistencia en bacterias de origen ambiental salvo los hechos anteriormente por nosotros $(12,13)$. Considerando el impacto que tienen las IIH en la economía de los pacientes y sus familias, sobre todo cuando son producidas por organismos resistentes a antimicrobianos, $y$ teniendo en cuenta que el adecuado seguimiento de estos gérmenes puede contribuir a controlar y disminuir la incidencia, se realizó el estudio con el objetivo de identificar enterobacterias contenidas en reservorios intrahospitalarios del Hospital Regional de Cajamarca, evaluándose su sensibilidad frente a antibióticos betalactámicos y su capacidad para producir BLEE como mecanismo de resistencia.

\section{MATERIAL Y MÉTODOS}

\section{Toma de Muestras}

Durante el último trimestre del año 2009 se obtuvieron un total de 55 muestras a partir de potenciales reservorios bacterianos (lavatorios, grifos, mesas, camas y tablillas de historia clínica), en salas con pacientes o vacías, incluyendo servicios higiénicos, pasillos y salas de vestir del personal, de las áreas hospitalarias de cirugía y pediatría del Hospital Regional de Cajamarca. Para la toma de muestra se humedecieron hisopos estériles en solución salina estéril; luego por el método de hisopado de superficies fueron rotándose sobre la superficie a muestrear; luego fueron introducidos en tubos con caldo infusión-cerebrocorazón para ser transportados en cadena de frío al Laboratorio de Microbiología de la Universidad Nacional de Cajamarca para su procesamiento inmediato.

\section{Aislamiento e identificación de Enterobacteriacea}

En el laboratorio, se retiró el hisopo y se incubó el caldo durante $24 \mathrm{~h}$ a $37^{\circ} \mathrm{C}$. Cumplido el tiempo, se sembró por estrías en agar sangre y McConkey y se incubó por $24 \mathrm{~h}$ a $37^{\circ} \mathrm{C}$. Se confirmó el crecimiento y se realizó coloración de Gram para verificar la morfología y pureza de los cultivos. En caso de encontrar más de un tipo de colonia se procedió a verificar si había o no contaminantes (gérmenes que no correspondan a la muestra), si se concluía que no había contaminantes se procesó como otro cultivo. Determinada la morfología y pureza de los cultivos, se realizó la prueba de oxidasa (Oxoid) para diferenciar
Enterobacteriaceae (negativos) de bacilos gram negativos no fermentadores (positivos), luego se continuó con la identificación bioquímica por métodos convencionales (1).

\section{Prueba de sensibilidad a antibióticos betalactámicos}

La prueba de sensibilidad a betalactámicos se realizó con el método de disco difusión y su interpretación con los puntos de corte del comité europeo para pruebas de susceptibilidad (14). Los betalactámicos probados (Oxoid) fueron: ampicilina (AMP), cefalotina (KF), cefoxitina (FOX), amoxicilina/acido clavulánico (AMC), aztreonam (ATM), imipenem (IPM), ceftazidime (CAZ), cefotaxime (CTX) y ceftriazone (CRO). Las cepas resistentes a una o varias C3G y positivas al tamizaje (4), pasaron a la prueba confirmatoria para BLEE.

\section{Determinación de producción de BLEE}

Se empleó el método del doble disco descrito por Drieux (15); para ello, se prepararon suspensiones bacterianas en solución salina fisiológica estéril al 0,85\% con turbidez comparada al tubo 0,5 MacFarland y luego se inocularon en agar Mueller-Hinton (Merck). La prueba consistió en colocar un disco de AMC (20/10 $\mu \mathrm{g})$ en el centro de las placas, y alrededor de éste se colocaron, a una distancia de $20 \mathrm{~mm}$, discos de CAZ $(30 \mu \mathrm{g})$, CTX $(30 \mu \mathrm{g})$, CRO $(30 \mu \mathrm{g})$ y ATM $(30 \mu \mathrm{g})$. Las placas fueron luego incubadas por 24 horas a $37^{\circ} \mathrm{C}$. La presencia de BLEE se confirmó por el efecto sinérgico del inhibidor (efecto tapón de corcho en la figura 1), en la forma de ampliación del halo en uno o varios de los betalactámicos incluidos en la prueba (2).

\section{Control de calidad}

Para el control de calidad de las pruebas se utilizaron las cepas ATCC 700603 de K. pneumoniae productora de BLEE y la cepa no productora ATCC 25922 de E. coli.

\section{Análisis estadístico}

En el análisis estadístico, para determinar la frecuencia de positividad de enterobacterias en los reservorios y de BLEE en los cultivos, se empleó el programa SPSS v15. Se empleó el chi cuadrado para estimar la asociación entre las áreas hospitalarias y los cultivos productores de BLEE, considerándose estadísticamente significativo un $\mathrm{p}<0,05$. 


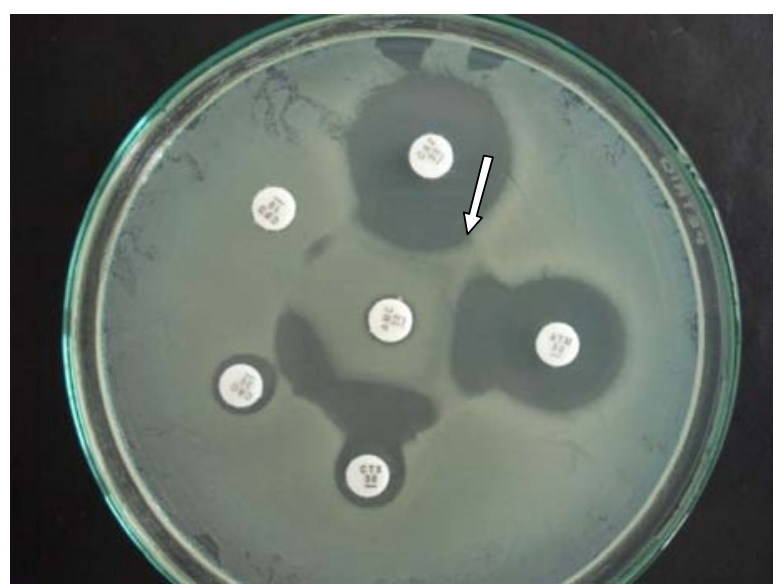

Figura 1. Efecto sinérgico de CAZ, ATM, CTX y CRO con AMC en la prueba confirmatoria de producción de BLEE en una cepa de Enterobacter cloacae.

\section{RESULTADOS}

A partir de las muestras se recuperaron, aislaron e identificaron 45 cultivos de enterobacterias de importancia clínica: 14 E. cloacae, 11 E. coli, 5 C. freundii, 4 K. pneumoniae y 11 de otros géneros (Tabla 1). En veinte muestras no se obtuvo aislamiento; de los 45 aislamientos obtenidos, 25 fueron hallados como únicos en el respectivo reservorio, y diez reservorios presentaron hasta dos cultivos de enterobacterias diferentes (Tabla 2).

La susceptibilidad de las enterobacterias aisladas a los betalactámicos, se muestra en el gráfico 1 .

Tabla 1. Distribución de cultivos de Enterobacteriaceae según reservorio y BLEE positivos.

\begin{tabular}{lccccccc}
\hline \multirow{2}{*}{ Microorganismo } & \multicolumn{8}{c}{ Reservorios o superficie muestreada } & \multirow{2}{*}{ Totales } & \multirow{2}{*}{ BLEE+ } \\
& Grifo & Lavatorio & Cama & Mesa & Tablilla & & \\
\hline Enterobacter cloacae & - & 1 & 4 & 7 & 2 & 14 & 4 \\
Escherichia coli & 1 & 1 & 5 & 4 & - & 11 & 4 \\
Citrobacter freundii & 1 & 1 & - & 2 & 1 & 5 & - \\
Klebsiella pneumoniae & 1 & - & - & 3 & - & 4 & 1 \\
Hafnia alvei & - & - & 2 & 1 & - & 3 & - \\
Serratia marcescens & - & - & - & 1 & 1 & 2 & - \\
Yersinia sp. & - & - & - & 1 & 1 & 2 & 1 \\
Providencia stuartii & - & - & - & 1 & - & 1 & - \\
Enterobacter aerogenes & - & 1 & - & - & - & 1 & 1 \\
Proteus vulgaris & 1 & - & - & - & - & 1 & 1 \\
Salmonella sp. & - & - & - & 1 & - & 1 & - \\
Totales & $\mathbf{4}$ & $\mathbf{4}$ & $\mathbf{1 1}$ & $\mathbf{2 1}$ & $\mathbf{5}$ & $\mathbf{4 5}$ & $\mathbf{1 2}$ \\
\hline
\end{tabular}

Tabla 2. Frecuencia de reservorios con Enterobacteriaceae según áreas estudiadas.

\begin{tabular}{lcc}
\hline Variable & $\begin{array}{c}\text { Cirugía } \\
(\mathbf{n}=33)\end{array}$ & $\begin{array}{c}\text { Pediatría } \\
(\mathbf{n}=\mathbf{2 2})\end{array}$ \\
\hline Total de aislamientos & 22 & 23 \\
1 aislamiento por reservorio & 12 & 13 \\
aislamientos por reservorio & 05 & 05 \\
Reservorios con cultivo negativo & 16 & 04 \\
Aislamientos BLEE positivos* & 07 & 05 \\
\hline
\end{tabular}

$\mathrm{n}$ : número de muestras por área hospitalaria.

$* \mathrm{p}>0.05$ 
Gráfico 1. Sensibilidad a betalactámicos en Enterobacteriaceae aisladas.

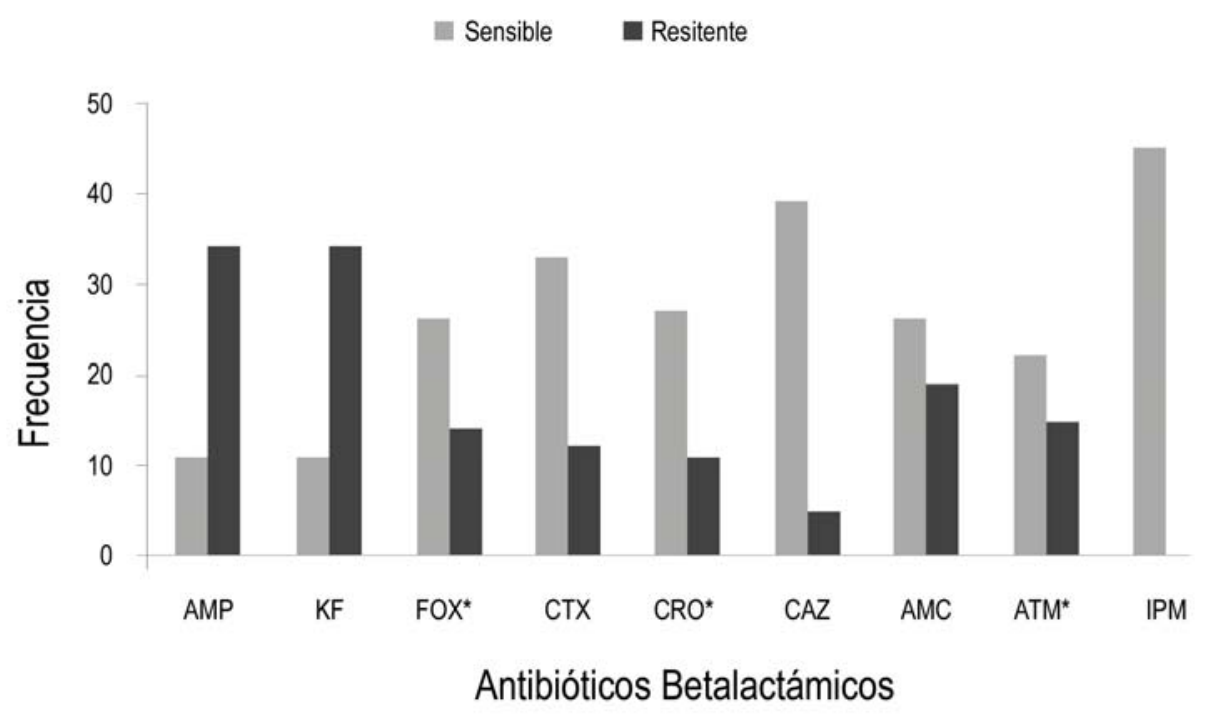

*No todos los aislamientos fueron evaluados

Doce cultivos (Tabla 1) fueron productores de BLEE, 4 de E. coli y 4 E. cloacae fueron los más resaltantes, lo que implica que son resistentes a $\mathrm{C} 3 \mathrm{G} \mathrm{o}$ monobactámicos; sin embargo, como es característico también de las BLEE, todos estos cultivos fueron sensibles a carbapenémicos (IPM). No se encontró diferencia en el número de cultivos productores de BLEE entre las áreas hospitalarias estudiadas ( $\mathrm{p}>0.05)$.

\section{DISCUSIÓN}

La aparición de cepas resistentes y multirresistentes constituye uno de los mayores problemas en los hospitales de todo el mundo, ya que dificultan el tratamiento de las enfermedades infecciosas y deterioran la economía y la calidad de vida de las personas (2). En ese sentido, las altas frecuencias de resistencia a los diferentes tipos de betalactámicos, significarían la presencia de uno o varios mecanismos de resistencia en las bacterias aisladas, cuyos fenotipos se evalúan mejor sobre la base de una adecuada interpretación del antibiograma, así por ejemplo, la resistencia a FOX está fuertemente relacionada con la presencia de betalactamasas cromosómicas tipo AmpC y la resistencia a C3G o monobactámicos (CAZ, CTX, CRO o ATM respectivamente) con la presencia de BLEE (16).
Todas las especies listadas en la tabla 1 han sido reportadas en múltiples trabajos de investigación como causas de IIH, con alta incidencia sobre todo de $E$. coli, Enterobacter sp. y K. pneumoniae $(2,6)$; aunque pocos estudios proveen evidencia convincente de que los organismos del ambiente sean fuente importante de trasmisión entre los pacientes, Bhalla et al. demostraron que algunos pueden ser adquiridos desde superficies contaminadas (8).

Las BLEE, que derivan principalmente de las clásicas enzimas TEM y SHV, no sólo son enzimas capaces de hidrolizar penicilinas, C3G y monobactámicos sino que localizadas en plásmidos son transferibles entre diversas especies bacterianas, lo cual agrava la situación. La prevalencia de Enterobacteriaceae resistentes está en aumento en muchos hospitales del mundo, principalmente E. coli y K. pneumoniae (16), pero esta resistencia se convierte en un problema mayor si originan brotes intrahospitalarios de identificación tardía y si desencadena multirresistencia antibiótica (17).

Los dispositivos y superficies de las áreas hospitalarias, pese a que se les ha subestimado como reservorios microbianos, se han visto afectados por la propagación de microorganismos ubicuos, y pueden ser origen de brotes importantes de IIH $(7,8,11)$. Según 
el tipo de reservorio, hallamos que la mayor proporción de cultivos BLEE $(+)$ provenía de camas y lavatorios sugiriendo una constante ya que antes habíamos reportado la presencia de otras bacterias de importancia patógena, también con capacidad de producir algún tipo de betalactamasa en estos mismos reservorios $(12,13)$. Teng et al. (9) refuerzan la teoría de los reservorios de enterobacterias multidrogorresistentes al demostrar que aislamientos de $K$. pneumoniae de tablillas de historia clínica presentaban el mismo fenotipo de sensibilidad en el antibiograma que cultivos de la misma especie aislados de los pacientes.

El hallazgo de bacterias resistentes en superficies y reservorios intrahospitalarios podría reportarse de forma creciente en los próximos años, gracias a que muchas tienen la propiedad de unirse ávidamente a superficies y objetos hospitalarios (12), convirtiendo los ambientes hospitalarios en un sistema de contaminación bacteriana que puede llegar a desencadenar IIH, cuyo principal vehículo de trasmisión serian las manos del personal que trabaja en el hospital $(9,11)$. De las especies halladas, E. coli, $K$. pneumoniae, dos de las principales especies productoras de BLEE, y E. cloacae han sido asociadas con brotes intrahospitalarios por cepas resistentes, lo cual justificaría una asociación causal de IIH que puede complicarse cuando es originada por microorganismos multirresistentes. Además $C$. freundii, S. marcescens y E. aerogenes también pueden producir BLEE, y algunas cepas pueden generar más de un tipo de enzima $(15,18)$.

Aunque esta investigación no provea evidencia de trasmisión a los pacientes, nuestros datos sugieren que el ambiente puede contribuir significativamente a la contaminación de las manos con microorganismos multirresistentes, donde por obvias razones los trabajadores de salud podrían jugar un papel importante como vehículos de trasmisión de éstos potenciales patógenos. Exponemos también como una necesidad el seguimiento de estas bacterias, de origen no clínico, productoras de BLEE, basado en cuatro razones principales: el potencial de trasmisión de estas cepas, los brotes intrahospitalarios que pueden desencadenar, el uso excesivo de antibióticos de amplio espectro que su hallazgo significa y el reto de limitar los brotes epidémicos controlando las resistencias y multirresistencias.

Estos resultados, además, refuerzan la importancia de una apropiada limpieza y desinfección de las superficies y sobre todo del lavado de manos, sobre todo después del contacto con reservorios potencialmente contaminados. Este es probablemente, en nuestro país, el primer reporte de enterobacterias aisladas de superficies o reservorios intrahospitalarios con demostrada capacidad de producir BLEE.

Es necesario indicar algunas de las limitaciones más importantes de este trabajo. No se determinó la densidad de las bacterias halladas en los reservorios, dato importante porque con densidades pequeñas o bajas de los gérmenes se podría reducir significativamente el potencial de trasmisión. Por otro lado, la comparación con cepas de origen clínico podría habernos permitido establecer una relación más fuerte con las $\mathrm{IIH}$, estudio que se debe implementar en el futuro. La prueba de screening empleada para la detección de BLEE productores tiene sus limitaciones, por lo que se pudieron haber perdido aislamientos positivos. Finalmente, no determinamos los fenotipos específicos de las cepas BLEE positivas, por la diversidad de estas enzimas, que en la actualidad es muy amplia y además, la tarea es logísticamente muy costosa, ya que muchos de estos mecanismos de resistencia sólo son detectables por procedimientos de tipificación molecular.

\section{Agradecimientos}

Expresamos nuestro agradecimiento a la Unidad Coordinadora del Programa de Ciencia y Tecnología de la PCM y al CONCYTEC por los recursos asignados al autor principal (contrato $\mathrm{N}^{\mathrm{o}} 158-2009-\mathrm{FINCyT-BDN)}$ y que como parte de la tesis doctoral "Susceptibilidad a betalactámicos y caracterización de betalactamasas en cultivos de Enterobacteriaceas ambientales del Hospital Regional de Cajamarca, octubre 2009 - junio 2010", han permitido solventar buena parte de éste trabajo. También agradecemos al Dr. Nicolás López Chegne, Jefe del Departamento de Patología Clínica del Hospital Regional de Cajamarca por el apoyo desinteresado en la realización de éste trabajo.

Fuente de financiamiento: El autor principal es becario de la Unidad Coordinadora del Programa de Ciencia y Tecnología de la PCM con contrato $\mathrm{N}^{\mathrm{o}} 158$ 2009-FINCyT-BDN.

\section{Conflictos de interés: Ninguno}

\section{Correspondencia:}

Marco A. Rivera Jacinto.

Departamento de Ciencias Biológicas, Facultad de Ciencias de la Salud, Universidad Nacional de Cajamarca. 
Dirección: Ciudad Universitaria. Av. Atahualpa 1050.

Edificio 1D. Oficina 105. Cajamarca

Correo electrónico: mrivera@unc.edu.pe

\section{REFERENCIAS BIBLIOGRÁFICAS}

1. Winn WC, Allen SD, Janda WM, et al. Koneman Diagnóstico microbiológico. $6^{\mathrm{a}}$ ed. Buenos Aires: Editorial Médica Panamericana; 2008. p. 205.

2. Perozo AJ, Castellano MJ. Detección de betalactamasas de espectro extendido en cepas de la familia Enterobacteriaceae. Kasmera 2009; 37(1):25-37.

3. Villegas MV, Kattan JN, Quinteros MG, Casellas JM. Prevalence of extended-spectrum â-lactamases in South America. Clin Microbiol Infect 2008; 14(S1):154-158.

4. Seral C, Pardos M, Castillo F. Betalactamasas de espectro extendido en enterobacterias distintas de Escherichia coli y Klebsiella. Enferm Infecc Microbiol Clin 2010; 28(S1):12-18.

5. Suárez C, Gudiol F. Antibióticos betalactámicos. Enferm Infecc Microbiol Clin 2009; 27(2):116-129.

6. Ramón P, Fernández-Cruz A. Magnitud y tendencia de la resistencia a los antibióticos de gérmenes gramnegativos hospitalarios en América Latina. Rev Panam Infectol 2008; 10(4 Suppl. 1):S38-S46.

7. Hota B. Contamination, disinfection, and crosscolonization: are hospital surfaces reservoirs for nosocomial infection? Clin Infect Dis 2004; 39:11821189.

8. Bhalla A, Pultz NJ, Gries DM, et al. Acquisition of nosocomial pathogens on hands after contact with environmental surfaces near hospitalized patients. Infect Control Hosp Epidemiol 2004; 25:164-167.

9. Teng SO, Lee WS, Ou TY, Hsieh YC, Lee WC, Lin YC. Bacterial contamination of patients' medical charts in a surgical ward and the intensive care unit: impact on nosocomial infections. J Microbiol Immunol Infect 2009; 42:86-91.

10. Kramer A, Schwebke I, Kampf G. How long do nosocomial pathogens persist on inanimate surfaces?
A systematic review. BMC Infectious Diseases 2006; 6:130.

11. Pittet D, Allegranzi B, Sax H, et al. Evidence based model for hand transmission during patient care and the role of improved practices. Lancet Infect Dis 2006; 6:641-652.

12. Rivera-Jacinto M, Rodríguez-Ulloa C, Huayán-Dávila G. Pseudomonas aeruginosa productora de betalactamasa clásica y de espectro extendido en reservorios de un servicio de neonatología. Rev Peru Med Exp Salud Publica 2008; 25(2):250-252.

13. Rivera-Jacinto $M$, Rodríguez-Ulloa C, Huayán-Dávila G. Frecuencia de aislamientos ambientales de Staphylococcus aureus y su actividad beta-lactamasa en un hospital de Cajamarca, Perú. Infectio 2009; 13(3):192-195.

14. European Committee for Antimicrobial Susceptibility Testing. Breakpoint tables for interpretation of MICs and zone diameters. Version 1.3, January 5, 2011. URL disponible en:http://www.eucast.org/fileadmin/src/ media/PDFs/EUCAST_files/Disk_test_documents/ EUCAST_breakpoints_v1.3_pdf.pdf. (Fecha de acceso: 12 de febrero 2011).

15. Drieux L, Brossier F, Sougakoff W, Jarlier V. Phenotypic detection of extended-spectrum â-lactamase production in Enterobacteraceae: review and bench guide. Clin Microbiol Infect 2008; 14(S1):90-103.

16. Livermore DM, Winstanley TG, Shannon KP. Interpretative reading: recognizing the unusual and inferring resistance mechanisms from resistance phenotypes. J Antimicrob Chemother 2001; 48(S1):87102.

17. Okesola AO, Makanjuola O. Resistance to ThirdGeneration Cephalosporins and Other Antibiotics by Enterobacteriaceae in Western Nigeria. Am J Infect Dis 2009; 5(1):17-20.

18. Bertona E, Radice M, Rodríguez CH, et al. Caracterización fenotípica y genotípica de la resistencia enzimática a las cefalosporinas de tercera generación en Enterobacter spp. Rev Argent Microbiol 2005; 37:203-208.

Recibido: 02/11/2010

Aceptado para publicación: 12/05/2011 